\title{
FIRST REPORT OF CORONARY VASOSPASM WITH SERTRALINE IN CHILDREN
}

\author{
Irfan Oguz Sahin \\ Ondokuz Mayls University, Department of Pediatrics, Division of Pediatric Cardiology, Samsun, Turkey \\ received: 25.10.2020; \\ revised: 5.1.2021; \\ accepted: 26.1.2021
}

\section{INTRODUCTION}

Drugs acting primarily on central nervous system have potential side effects on cardiovascular system (CVS). There is a recent interest in cardiotoxicity of these drugs (Waring 2012).

Sertraline (SRT), a commonly prescribed selective serotonine reuptake inhibitor (SSRI), is known to have safer cardiotoxicity profile than tricyclic antidepressants (TCAs). The most common cardiovascular effects of SRT are bradycardia, QT prolongation, alteration of T wave, hypotension and syncope (Pacher et al. 2004). According to our literature review, this is the first case of coronary vasospasm with SRT in an adolescent.

\section{CASE REPORT}

A previously healthy 14-year-old male admitted to hospital with chest pain. Sertraline was started 20 days ago in a dose of $50 \mathrm{mg} /$ day for anxiety and phonophobia and 3 days ago sulbactam/ampicillin (SAM) was started for upper respiratory tract infection (URTI). Past medical history was unremarkable for the patient and his family.

Vital signs and physical examination were normal. Initial ECG showed elevated ST segments in DI-II, aVL, V4-6 leads suggestive of lateral ischemia (Figure 1). Chest X-ray and transthoracic echocardiogram (TTE) were normal. Cardiac enzymes were markedly elevated [CK-MB: $108 \mathrm{ng} / \mathrm{mL}$ (range 0-0.48), troponin I (TnI) $>25 \mathrm{ng} / \mathrm{mL}$ (range 0-0.11)].

Myopericarditis or coronary vasospasm likely secondary to SRT were considered as possible etiologies. Naproxen, captopril, aspirin, metoprolol were started and SRT was discontinued. Within 24 hours, ST segment started to get normal and completely normalized at the $3^{\text {rd }}$ day. Serum TnI levels showed a downward trend and returned to normal levels on the $5^{\text {th }}$ day. CK-MB started to decrease at the $1^{\text {st }}$ day but increased at the $36^{\text {th }}$ hour and then returned to range within 5 days. He was discharged home as the cardiac enzymes returned to normal.

\section{DISCUSSION}

There is a recent concern about serious cardiovascular events (SCVE) with psychotropic drugs (Naguy et al 2016). In literature, some reports about minor CVS events and a few about sudden cardiac death are present (Houghton et al. 2019). Until the reporting of SRTassociated cardiotoxicities in the early 2000s, SSRIs were accepted to be safe (Pacher et al. 2004). Since then, there have been conflicting results on the cardiotoxicity of this drug. There is evidence that SSRIs have the potential for mild bradycardia, insignificant prolongation of QT intervals, dysrhythmia, syncope, infrequent hypertension and postural hypotension (Ilgin et al. 2018).

Initial TnI of our case was extremely high. The most common causes of high TnI levels in children are myocardial problems. Myocardial injury is a very rare adverse event of SRT. It should be observed due to increased oxidants and decreased (glutathione) antioxidants (Ilgin et al. 2018). Coronary vasospasm is very rarely reported with psychotropic drugs (Sinha et al. 2016). Another myocardial disease related is Tako-tsubo cardiomyopathy (TCM) which is the result of coronary vasospasm due to the enhanced activity of catecholamine by SSRI (Naguy et al. 2016). Characteristics of TCM are left ventricle wall hypokinesis, ballooning of left ventricle apex and inferolateral ST elevation.

ECG is a main tool to evaluate cardiotoxicity (Ilgin et al 2018). While TCAs pose the greatest risk for QT prolongation, SRT was shown to prolong QTc too (Rochester et al. 2018). PR, QTc ( $<0.45 \mathrm{~ms})$, T-wave amplitude values of our case were normal but ST segments were elevated at the DI-II, aVL, V4-6 leads. Myocarditis shows generalized changes on ECG while myocardial ischemia is prone to reveal localized findings (Pozo et al. 2014). The ECG of case showed ST change localized to lateral leads suggesting a lateral myocardial problem. The other main tool to differ myocardial and pericardial problems is TTE. Pericardial effusion, local and generalized hypokinesis are indicatives of pericarditis, myocard ischemia and myocarditis, respectively. Short-term hypoperfusion of myocardium as seen with coronary vasospasm would reveal a normal TTE. Also, a subset of adolescents with a family history of hypercholesterolemia or myocardial infarction can have myocardial infarction, our case lacked these risk factors and had no pathologic TTE finding (Dadfarmay et al. 2009). Myocarditis, pericarditis and myocardial infarction was excluded with TTE in our case. Regarding to increased TnI, ST elevation in lateral leads and normal TTE findings, the most likely diagnosis was left anterior descending coronary vasospasm. 

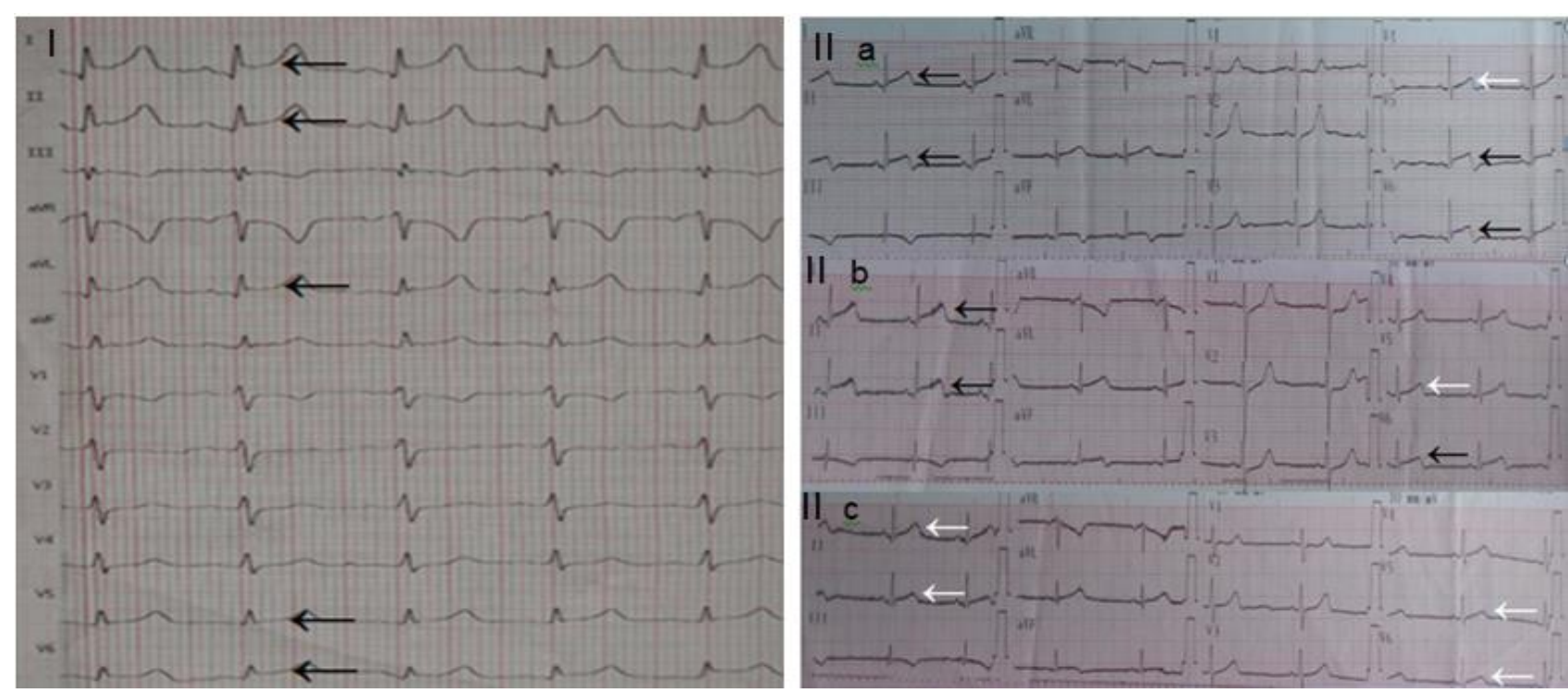

Figure 1. I. Initial ECG with ST segment elevation in DI-II, aVL, V4-6 leads (black arrows); II. Serial ECG findings at the $24^{\text {th }}$ (a), $48^{\text {th }}$ (b) and $72^{\text {th }}$ hours (c) show normalizing ST segments (white arrows)

Adverse reaction type and severity is related with individual and environmental factors. Usually, overdoses of SRT result SCVEs (Naguy et al. 2016). Studies showed that SRT prolongs QT significantly only when it is taken with other QT prolonging drugs.

The patient was started SRT 20 days and SAM 3 days before admission. Coincidence of the chest pain, URTI and SAM treatment suggest a relation between SRT, coronary vasospasm, infection and SAM. It is not possible to clarify this situation with current medical practices but we think that it would be useful to advise the patients to whom SRT were started to be carefull for chest pain especially in case of an URTI and antibiotic use. In this case, we noticed that $\mathrm{TnI}$ and ECG are more reliable parameters in follow-up of the disease than CKMB due to the increase of CK-MB at the $36^{\text {th }}$ hour instead of normalizing process of ECG and TnI.

\section{CONCLUSION}

Our case is important in term of being the first case of coronary vasospasm with SRT in children. Patients and families should be advised to admit to hospital in case of chest pain which should be associated with coronary vasospasm. Clinicians should also keep in mind that URTI and/or SAM should increase coronary vasospasm risk with SRT even with normal doses. Further microscopic, toxicologic and biochemical investigations are needed to clarify cardiotoxicity of SRT in children.

Informed consent was obtained for publication from the patient's parents.
Acknowledgments: None.

Conflict of interest: None to declare.

\section{References}

1. Dadfarmay S, Dixon J: A Case of Acute Cardiomyopathy and Pericarditis Associated with Methylphenidate. Cardiovasc Toxicol 2009; 9:49-52

2. Houghton R, de Vries F, Loss G: Psychostimulants/atomoxetine and serious cardiovascular events in children with $A D H D$ or autism spectrum disorder. CNS Drugs 2020; 34:93-101

3. Ilgin S, Kilic V, Baysal M, Aydogan-Kilic G, Ucarcan S, Dermenci B, et al: Evidence for cardiotoxicity associated with sertraline in rats. Toxicol Res (Camb) 2018; 7:817-25

4. Naguy A, Al-Mutairi H, Al-Tajali A: Atomoxetine-related Takotsubo Cardiomyopathy. J Psychiatr Pract 2016; 22:232-3

5. Pacher P, Kecskemeti V: Cardiovascular side effects of new antidepressants and antipsychotics: new drugs, old concerns? Curr Pharm Des 2004; 10:2463-75

6. Pozo E, Sanz J: Differentiating infarction from myocarditis. Heart Metab 2014; 62:13-17

7. Rochester MP, Kane AM, SA, Fixen DR: Evaluating the risk of QTc prolongation associated with antidepressant use in older adults: a review of the evidence. Ther Adv Drug Saf 2018; 9:297-308

8. Sinha A, Lewis O, Kumar R, Yeruva SL, Curry BH: Amphetamine abuse related acute myocardial infarction. Case Rep Cardiol 2016; 2016:7967851. doi:10.1155/2016/7967851

9. Waring WS: Clinical use of antidepressant therapy and associated cardiovascular risk. Drug Healthc Patient Saf 2012; 4:93-101

\section{Correspondence:}

Irfan Oguz Sahin, MD

Division of Pediatric Cardiology, Department of Pediatrics, Faculty of Medicine, Ondokuz Mayls University

Samsun, Turkey

E-mail:rfnshn@yahoo.com 